\title{
25 Research Square \\ Effects of Education on Symptom Recognition and Management of Pseudobulbar Affect: A Mixed- Methods Evaluation of Clinician Knowledge and Behavior Changes
}

\section{Wendy Turell}

PlatformQ Health Education

Anne Roc

PlatformQ Health Education

Erik Pioro

Cleveland Clinic

Alexandra Howson ( $\square$ alexhowson@thistleeditorial.com )

Thistle Editorial LLC https://orcid.org/0000-0002-1740-2925

Research article

Keywords: Pseudobulbar affect; online education; mixed methods evaluation; professional education

Posted Date: July 11th, 2019

DOl: https://doi.org/10.21203/rs.2.11238/v1

License: (c) (i) This work is licensed under a Creative Commons Attribution 4.0 International License.

Read Full License 


\section{Abstract}

Background Pseudobulbar affect (PBA) is under-recognized and often undertreated in clinical practice. There are few resources to support clinicians in the recognition and treatment of PBA symptoms, despite the approval by the United States Food and Drug Administration of a PBA-specific treatment. We evaluated the impact of a virtual education symposium on clinician knowledge and competence designed to improve knowledge and skills associated with identifying and managing PBA in patients with neurologic injury. Methods We designed a mixed methods outcomes methodology that included surveybased methods and qualitative interviews to measure trends in knowledge and competence. We measured changes in knowledge and competence via pre- and post-test online surveys that included case-based competence questions and knowledge-focused questions. McNemar test compared matched pair responses. Effect size was computed using Cohen's d for all significant findings. Significance testing was conducted for matched pairs of learners who completed both pre/post and pre/survey, respectively. We explored the impact of the educational intervention on clinician knowledge, competency, and perspectives on PBA via qualitative interviews with a subsample of education participants. We used a process of constant comparison to structure analysis of participant responses to questions both across and within interviews. Results Participants' PBA symptom recognition knowledge and skills increased following program participation and were maintained at follow-up. Responses to post-activity case-based questions showed that exposure to education enabled participants to better recognize and describe symptoms redolent of PBA. Following education, most participants said they would initiate therapy with on-label versus off-label medications. Qualitative descriptions of what interviewees say they would do in clinical practice were reflected in actual responses to knowledge and competence questions. Conclusions This mixed-method study demonstrates that online education is an effective and accessible tool for building knowledge and supporting clinical competence concerning PBA symptom recognition and management.

\section{Background}

Pseudobulbar affect (PBA) is characterized by unprovoked outbursts of uncontrollable, inappropriate laughter and/or crying that are unrelated, exaggerated or out of proportion to the emotions felt by the patient or to the social context in which they are expressed. ${ }^{1-3}$ This debilitating condition affects an estimated 2 to 7 million people in the United States, ${ }^{2,4,5}$ and occurs secondary to neurological diseases such as Alzheimer's disease (AD), amyotrophic lateral sclerosis (ALS), Parkinson's disease (PD), multiple sclerosis (MS), stroke, and traumatic brain injury (TBI). ${ }^{6}$ The prevalence of PBA symptoms has been reported in patients with TBI ( 50\%), followed by MS, ALS, stroke (38\%), AD and PD. ${ }^{4}$ PBA symptoms are also associated with psychiatric disorders and depression, ${ }^{7}$ and are highly prevalent among military service members, in whom TBI and post-traumatic stress disorder are common. ${ }^{8}$ The symptoms of PBA confer a substantial emotional and social burden on patients and caregivers, often resulting in embarrassment, social withdrawal or isolation, as well as reduced work productivity and quality of life. ${ }^{9}$ Symptoms appear to be determined largely by the anatomic location of brain lesions, primarily in the 
frontal lobes and descending pathways to the brain stem, basis pontis, and cerebellum. These lesions disrupt cortico-pontine-cerebellar neural networks that regulate emotional expression, resulting in loss of supra-nuclear control. ${ }^{3-5}$ In addition to structural damage, neurotransmitters and neuromodulators, especially serotonin and glutamate, may be involved in the pathology of PBA., 3

PBA is under-recognized in clinical practice and symptoms are often attributed to psychiatric or mood disorders (e.g., depression), rather than to neurological etiology. ${ }^{2,6}$ There is confusion around the nosology and diagnostic criteria for PBA symptoms, such that patients with PBA tend not to report their symptoms and clinicians fail to ask about them. ${ }^{6,10}$ PBA is also often undertreated. ${ }^{2}$ Although off-label use of selective serotonin reductase inhibitors (SSRIs) have shown some efficacy in treating PBA symptoms, ${ }^{11}$ other off-label medications (e.g., tricyclic antidepressants) lack clinical evidence on their efficacy and safety. ${ }^{6}$ In contrast, dextromethorphan/quinidine (DM/Q) is approved by the United States Food and Drug Administration (FDA) for treating the neurocircuitry damage underlying PBA symptoms on the basis of results from three large-scale clinical trials. ${ }^{12,13}$ The American Academy of Neurology guidelines recommends that this agent be considered for treating PBA in patients with ALS and MS. ${ }^{10,14}$.

There are few resources to support clinicians in the recognition and treatment of PBA symptoms, despite the approval for a PBA-specific treatment. In order to develop and provide such resources, PlatformQ Health Education, LLC (PQHE), in conjunction with Albert Einstein College of Medicine and Montefiore University Hospital created an interactive online continuing medical education activity. We evaluated the impact of education on clinician knowledge and competence via a mixed methods outcomes methodology that included survey-based methods and qualitative interviews.

\section{Methods}

\section{Education Program Design and Delivery}

Documented knowledge gaps concerning PBA point to stigma and social isolation, under-reporting, and misdiagnosis of this debilitating condition. Based on this educational need, Cases, Challenges, and Care of Pseudobulbar Affect in Neurological Diseases was designed to improve knowledge and skills associated with identifying and managing PBA in patients with neurologic injury and apprise clinicians of recommended treatment options that are indicated for managing patients with a diagnosis of PBA. The target audience included neurologists, psychiatrists, geriatricians, primary care clinicians (internal, general and family medicine), and advanced health care providers (nurse practitioners [NPs] and physician assistants [PAs]) involved in the care of patients with neurological diseases who may be suffering from PBA. The live, virtual session was recorded in a production studio and broadcast live on NeuroSeriesLive.com on September 13, 2016. The program was subsequently available on-demand at the 
same website for 12 months through September 13, 2017. The live and on-demand education were certified for continuing medication education (CME) credit and allowed access to downloadable educational materials. The program content consisted on two faculty-panel members who discussed the impact of PBA in patients with neurological disease via case-based materials and reviewed how to recognize, evaluate and treat patients with PBA. Participants were able to submit questions before and during the live program, and faculty answered a sampling of these questions throughout the session. Live polling questions were also interspersed with faculty discussions and question responses and integrated into the discussion flow.

\section{Quantitative and Qualitative Evaluation of Knowledge, Competence, and Practice Change}

\section{Participants, Inclusion/Exclusion Criteria, and Recruitment}

Continuing medical education learners were invited to participate in this education activity via emails from educational databases housed at NeuroSeriesLive.com and Albert Einstein College of Medicine and Montefiore University Hospital. The education activity was also promoted through social media channels (Facebook, Linkedln, Twitter) and advertisements on google. We recruited potential interviewees from this larger sample of education program participants 6-10 weeks following activity participation. Participants were eligible to participate in interviews if they managed patients with PBA, currently resided in the USA, and recalled viewing the education program. We determined this eligibility via an invitation and screener emailed to education program participants. Those who did not meet the inclusion criteria were not invited to participate in interviews. When screening for potential interviewees began, 169 participants had completed the education activity and 79 responded to interview invitations. Of these invitation respondents, 31 were eligible as per inclusion criteria. A small honorarium was offered for completing an interview. Western Institutional Review Board granted ethical approval for the study and we obtained informed consent from all participants prior to interviews. None of the participants were personally known to the authors.

Quantitative Data. McNemar test compared matched pair responses (those who completed pre/post and pre/8wk). Effect size was computed using Cohen's $d$ for all significant findings (small $\geq .20$, medium $\geq$ .50 , large $\geq .80) .{ }^{19}$ Significance testing was conducted for matched pairs of learners who completed both pre/post and pre/survey, respectively. Graphed data that is presented in bar charts represents pooled data from pretest, posttest and survey completers, respectively (all responses, not limited to those who could be matched).

\section{Results}

Participants 
We conducted semi-structured interviews with a subsample of 20 clinicians (Table 1). Most participants typically saw patients with both neurodegenerative/neurocognitive and psychiatric conditions (e.g., depression, anxiety, schizophrenia, bipolar disorder). Neurologists more typically saw patients with neurologic/neurodegenerative conditions.

\section{Key Qualitative Themes}

\section{Awareness of PBA}

Few interviewees reported direct or sustained clinical exposure to patients with known PBA prior to education exposure and only a minority of participants (25\%) saw more than 16 patients with PBA per month. Despite little direct contact with PBA patients, interviewees were aware of PBA as a condition associated with neurologic conditions from various sources such as participating in prior CME activities, promotional education, recent residency, and direct experience of managing patients with PBA. Similarly, all interviewees were aware of the broad impact that PBA symptoms have on patients and their caregivers/families, including distress, embarrassment, social withdrawal, feelings of frustration and humiliation, and impaired social and occupational function.

\section{PBA Symptom Recognition}

Interviewees emphasized that PBA symptoms can be subtle and challenging to recognize (internal medicine doctors don't always recognize it). They were also aware of the varied nosology used to describe symptoms of PBA, such as dementia, psychosis, depression, agitation, 'emotional incontinence', pseudobulbar palsy, pathological laughing or crying, or post-stroke syndrome. Interviewees generally acknowledged that over time they had probably missed opportunities to diagnose patients with PBA. Even among neurologists in the sample reflected that PBA was not always 'top of mind'.

I definitely would have attributed it to something else. I think that the diagnosis of PBA really doesn't immediately come to the forefront for us. [Provider 19, neurologist, academic center]

I think I might have heard about it, but I never paid that much attention to it. I never really thought about it at all...you certainly didn't screen for it or pay much attention to it. It was mostly off radar or you'd think like maybe some psychiatric issues there. [Provider 2, internal medicine, group practice]

Following education, all interviewees correctly described PBA symptoms as a constellation of involuntary and spontaneous emotional outbursts consisting of laughing or crying, that may be sudden, brief, and incongruent or exaggerated relative to patient's underlying mood. Although it is possible that interviewees were familiar with these symptoms prior to education exposure, they also highlighted symptom recognition as a key element of knowledge building delivered by the education activity. 
It really helped to decipher the difference between those patients that are maybe just the depression and anxiety and then just delving into the symptoms that separate PBA, and kind of being able to separate out those patients and give them the right diagnosis. [Provider 5, physician assistant, general practice]

It heightened my awareness of the diagnosis. [Provider 19, neurology, academic]

\section{Education Impact on Knowledge about Causes of PBA}

When asked which conditions most raised suspicion for PBA, more than half of interviewees identified $T B I$ as a leading condition associated with PBA, followed by AD/PD, stroke, MS, and ALS (Table 2). These estimates differ somewhat from PRISM study prevalence but may reflect prevalence of the conditions in their patient populations. ${ }^{4}$

When asked to describe the causes of PBA, half of the interviewees referred to elements of its complex pathophysiology and half identified the most common neurological conditions associated with PBA as causal explanations. These descriptions are consistent with content provided in the education activity.

Pathophysiological Descriptions of the Causes of PBA:

There's a breakdown in communication between the neurotransmitters, the parts of the brain that actually controls emotions. [Provider 10, geriatrics, private practice]

Like on the webinar thing that I watched you know, they talked about there's some that aren't clear, but it's some kind of disruption in the brain system, I believe it was the cerebro-ponto-cerebellar circuit. [Provider 11 , nurse practitioner, group practice]

I would describe it as a wiring problem between the prefrontal cortex and the cerebellum. The wiring goes wrong in some patients basically with traumatic brain injuries or with Alzheimer's or MS or stroke or even Parkinson's disease. [Provider 13, family medicine, group practice]

Neurological Descriptions of the Causes of PBA: 
I think those are the big ones in addition to the other ones like multiple sclerosis, ALS and I think stroke. [Provider 2, internal medicine, group practice]

Like I said, the traumatic brain injury, the injury you know to the back of the head. A lot of the stroke victims. We do have MS patients who have symptoms like the dementia or Parkinson's or Alzheimer's, people like that. [Provider 9, nurse practitioner, group practice]

I'm learning now that certainly whatever biochemical changes may be happening in the brain, especially after a stroke, for example, may be contributing to it. [Provider 19, neurology, academic]

\section{Differentiating PBA from Depression or Other Conditions}

We asked interviewees to describe which symptoms or characteristics would differentiate PBA from other conditions. Responses fell into 3 categories that all represent content addressed in the education activity (Table 3). Spontaneous or exaggerated emotions was the most frequently cited differentiating feature of PBA across interviewees. Neurologists typically contrasted the brief duration of PBA symptoms versus the longer lasting symptoms associated with depression or other conditions. Neurologists and primary care clinicians also pointed to the presence of a neurological diagnosis or neurodegenerative condition as a key differentiating feature of PBA versus depression or other conditions.

Interviewees with lowest clinical exposure to PBA (i.e. reporting 1-5 patients with PBA in their practice) were more likely to identify spontaneous expression as the key differentiating feature of PBA vs depression (Figure 1).

\section{Education Impact on Knowledge about Diagnosis and Evaluation}

Clinical history is key in evaluating patients for PBA and reaching a diagnosis involves asking several questions to differentiate PBA from other conditions. Although interviewees were unaware of diagnostic criteria to confirm a diagnosis of PBA, most emphasized the importance of clinical history as their starting point for evaluation. Half said that it was important to involve caregivers where appropriate and available and neurologists (35\%) reported that, following clinical history, they would also order either a brain MRI or CT scan for patients to identify underlying neurodegenerative disease. The group was broadly divided in terms of whether their evaluation questions should emphasize the clinical features of 
PBA versus questions to identify an underlying pathology associated with PBA or other approach (Additional File 2). There was no pattern in the emphasis according to interviewee specialty.

Depression was the condition that most interviewees (80\%) identified as a differential diagnosis for PBA, followed by bipolar disorder $(10 \%)$ and mood disorders or other causes $(10 \%)$. All interviewees were able to identify one or more of the following clinical features that are key in differentiating PBA from depression:

- Uncontrollable crying, laughing or both

- Underlying neurologic disease/brain injury

- Brief duration of emotion expression

- Exaggerated or incongruent feelings

- Disconnect of emotional expression from underlying thoughts.

The Center for Neurologic Study - Lability Scale (CNS-LS) is a self-administered seven-item tool that has been validated for identifying PBA in patients with ALS and MS. ${ }^{20,21}$ Half of the interviewees commented that the education activity raised their awareness about the CNS-LS score.

I was unaware of the tools available for assessment. That CNS-LS, I didn't know that existed. That was like a simple and worthwhile tool that I will try to integrate. [Provider 3, MD, internal medicine]

Four interviewees reported already using this tool in clinical practice, and four interviewees were unaware of the score even following exposure to information about it during the education activity.

\section{Education Impact on Managing PBA Symptoms}

We asked interviewees to describe their first management steps for a patient in whom they suspect PBA. Interviewees were aware that antidepressants have limited efficacy in the treatment of PBA, although some commented that formulary constraints meant they would have to use these agents as their first step in treatment. A majority (75\%) reported they would prescribe DM/Q first for patients with PBA alone, on the grounds that this is currently the medication with an approved indication for PBA. 
I mean, we know that that's at least at the present time the only thing that's indicated for it...if I have a medication that I can stay on label, I prefer to go that route to begin with. [Provider 10]

I probably would start them on DM/Q-I was impressed [in] the studies that were reviewed. [Provider 3]

We now [have] FDA-approved treatment options. Previously, we were just using whatever off label. So that's the difference. [Provider 18, neurologist, private practice]

A majority (65\%) of interviewees reported they would add antidepressants or other mood stabilizers to DM/Q in patients with an established PBA diagnosis who also have a coexisting mood disorder. Others stated they would add DM/Q if patients were already being treated for a co-existing mood disorder or refer the patient for psychiatric evaluation.

One of the luxuries in Nuedexta is that if it's going to show benefit, you're going to see it in two to three weeks, whereas antidepressants are two to three months. So you know if there's a question of is it one diagnosis or the two of them comorbidly, I'll treat the PBA first. And if there's no response whatsoever, then chances are it's more of a primary depressive problem and then we'll go [on] an antidepressant. But if they have response, we're going to see it quickly. [Provider 15, neurology, private practice]

If the patient has a mood disorder, I start with a mood stabilizer first. If no response, then I start adding PBA treatment. [Provider 6, internal medicine, group practice]

\section{Quantitative Changes in Knowledge, Competence, and Practice}

Overall, 1,128 clinicians participated in the live and on demand education program on www.NeuroSeriesLive.com $(n=961)$. More than half of participants who completed the follow-up survey $(58 \%, n=59)$ reported the activity positively impacted patient experience or outcome and 31 write-in examples were provided. A majority of participants also reported the activity positively impacted clinical practice $(75 \%, n=60)$ with 48 write-in examples provided (Additional File 3$)$. Multiple clinical practice changes were reported to improve as a result of participating in this activity (Table $4, n=60$, multiple responses permitted, Additional File 4).

Changes in knowledge and competence (applying knowledge) were measured via paired comparisons of test questions pre-activity $(n=743)$, post-activity $(n=364)$, and follow-up at 8 weeks post-activity $(n=60)$. Responses show improvements in competence and knowledge concerning PBA diagnosis and treatment (Table 5, Additional File 5).

\section{Discussion}

This mixed-methods study suggests that online education can support changes in knowledge and competence and reinforce best practices in PBA symptom recognition and management. Interviewees 
were aware of PBA before education, but few had direct or sustained clinical exposure to patients with PBA. Lack of exposure to patients with PBA, as well as confusion around its nomenclature are factors in poor identification of PBA symptoms in clinical practice. ${ }^{2,6}$ Interviewees identified PBA symptom recognition and differentiation as the most challenging aspects of PBA evaluation and diagnosis; however, they also identified symptom recognition as a key learning fostered by the education program.

Following education, interviewees were mostly able to estimate PBA prevalence in distinct patient populations and describe causes of PBA that were consistent with content provided in the education activity. They were also able to correctly describe PBA symptoms using tools provided by the education. Responses to post-activity case-based questions showed that exposure to education also enabled participants to better recognize and describe symptoms redolent of PBA.

As a result of the challenges in differentiating PBA from other conditions, clinical history is key in evaluation and diagnosis. Pathological crying and depression are symptoms that can both be present in ALS, movement disorders and stroke, although symptoms of depression typically last longer than crying episodes in PBA, which are usually brief. ${ }^{3}$ Therefore, duration of symptoms is considered useful in distinguishing PBA from depression. Interviewees were able to identify one or more clinical features that are key in differentiating PBA from depression, including duration of symptoms and exaggerated emotions. Interviewees with lowest clinical exposure to patients with PBA were more likely to identify exaggerated emotions as the key differentiating feature of PBA versus depression. This trend suggests effective education about how to differentiate PBA from other conditions. Interviewees also described a range of questions they would ask patients that focused on clinical features, underlying pathology, and depression co-existence and the education activity raised awareness for many learners about using the CNS-LS scale. Interviewees clearly identified this scale as a new tool with potential utility in clinical practice. Finally, following education, most interviewees said they would initiate therapy with DM/Q before off-label medications, an emphasis that was also reflected in follow-up survey questions. This outcome stands in contrast to what we know about treatment in current clinical practice. For instance, in a recent survey of patients with PBA $(n=2,318), 52 \%$ of respondents who discussed their symptoms with clinicians were not prescribed any medications at all. ${ }^{2}$

Qualitative descriptions of what interviewees say they would do in clinical practice were reflected in actual responses to knowledge and competence questions. Knowledge and competence about the relationship between PBA and neurological conditions, how to recognize symptoms, and treatment with approved therapy improved from baseline immediately following the education and were mostly sustained 8-weeks after the education. Not only did overall participant responses to knowledge and competence questions improve over time from baseline to post-activity, but qualitative interview 
responses provided context for survey responses. Overall, interview responses reflected key messages conveyed in the education program.

\section{LIMITATIONS}

Participants self-selected to seek further information about PBA and may be atypical of clinicians who encounter patients with conditions associated with PBA in clinical practice. Additionally, we did not collect baseline qualitative data alongside baseline survey data, nor were we able to match interview responses with learner responses to knowledge and competence questions. Nonetheless, the value of a mixed-method approach is the addition of qualitative context for interpreting quantitative evaluation outcomes. In our study, quantitative education outcomes suggest that HCP education can improve PBA symptom recognition and diagnosis, and, in turn, lead to effective treatment initiation. Finally, the qualitative sample size was small, as is conventional in qualitative research. However, qualitative analysis does not typically require large sample sizes to yield meaningful insights across a range of perspectives. ${ }^{17}$

\section{Conclusions}

Online learning is expanding as an accessible and effective mechanism for delivering education, including CME. Several studies conclude that online CME, as measured via a variety of objective and subjective assessments (e.g., pre-post knowledge tests, semi-structured interviews, and written or online surveys), is an effective tool to improve clinician knowledge and competence and improve patient health outcomes. ${ }^{22-25}$ These studies also show that clinicians who participate in online CME activities are more likely to make evidence-based clinical choices than non-participants, change their behaviors, and sustain knowledge gains. This mixed-method study demonstrates that online education is an effective and accessible tool for building knowledge and supporting clinical competence concerning PBA symptom recognition and management. Our analysis suggests that participants' PBA symptom recognition knowledge and skills increased following program participation and were maintained at follow-up. Learners emphatically endorsed the relevance of the education, particularly the inclusion of case studies to draw out clinical presentations in otherwise complicated cases, and, as a psychiatrist interviewee noted, the education activity also reinforced what many participants felt they were already doing in their practice.

It confirmed a lot of what I was thinking, feeling doing, which is always a comfort and that's part of the reason we do CME. It's like [as] I'm doing this, am I doing everything I should be doing? Is my understanding correct? So for me, it was more of a confirmation of what I was already doing in a sense of, I probably could be better about doing the formal screening things, but really when they come to me, the docs are more interested in getting me to rule out the others. [Provider 16, MD, psychiatry] 
As the population ages and life expectancy lengthens, HCPs are likely to encounter patients with the broad variety of neurologic disorders underlying PBA in a variety of community settings. Ongoing education will be a necessary adjunct for ensuring that HCPs are equipped to exercise vigilance with patients who have conditions associated with PBA, recognize PBA symptoms, evaluate patients appropriately, and initiate therapies that are known to be effective.

\section{Abbreviations}

$A D$ : Alzheimer's disease

ALS: Amyotrophic lateral sclerosis

DM/Q: Dextromethorphan/quinidine

DTC: Direct-to-consumer

FDA: Food and Drug Administration

MS: Multiple sclerosis

NSA: National Stroke Association

PD: Parkinson's disease

PBA: Pseudobulbar affect

PRO: Patient-reported outcomes

SSRIs: Selective serotonin reductase inhibitors

TBI: Traumatic brain injury

\section{Declarations}

Ethical Approval and Consent to Participate. Western Institutional Review Board granted ethical approval for the study. All procedures performed in studies involving human participants were in accordance with the ethical standards of the institutional and/or national research committee and with the $1964 \mathrm{Helsinki}$ declaration and its later amendments or comparable ethical standards. We obtained and recorded informed consent from all participants prior to interviews.

Consent for Publication. Not applicable. 
Availability of Data and Material. We did not ask participants for consent to publicly share data and do not have permission from education participants to share data in publicly archived datasets.

Competing Interests. EP has received consulting fees from Avanir Pharmaceuticals.

Funding. Educational activities and evaluation were supported by unrestricted educational grants from Avanir Pharmaceuticals. The funding body had no role in the design of the study, the collection, analysis, and interpretation of data and in writing the manuscript.

\section{Authors' contributions}

All authors have read and approved the manuscript.

WT: Conceptualized and oversaw mixed methods research design; logistic management of subject screening, recruitment, informed consent and scheduling; collected and analyzed quantitative data; review and editing of qualitative and quantitative evaluation questions and manuscript.

AR: Educational program medical director, conceptualized and oversaw educational gap analysis and educational design, developer of pre-post online survey questions, review and editing of qualitative and quantitative evaluation questions and manuscript.

EP: PBA content expert advisor, developer of educational content (which is evaluated in this research study), review and editing of qualitative and quantitative evaluation questions and manuscript.

AH: Collected and analyzed qualitative interview data; drafted manuscript.

Acknowledgements. The research team is deeply grateful to all education participants, especially those who agreed to be interviewed for the qualitative part of this study. 


\section{References}

1. Rosen HJ, Cummings J. A real reason for patients with pseudobulbar affect to smile. 2007. Ann Neurol. 61;2: 92-96.

2. Work SS, Colamonico JA, Bradley WG, Kaye RE. Pseudobulbar affect: an under-recognized and under-treated neurological disorder. Adv Ther. 2011;28;7:86-601

3. Ahmed A, Simmons Z. Pseudobulbar affect: prevalence and management. 2013. Therapeutics Clin Risk Manag. 2013;9:483-489.

4. Brooks BR, Crumpacker D, Fellus J, Kantor D, Kaye RE. PRISM: a novel research tool to assess the prevalence of pseudobulbar affect symptoms across neurological conditions. PloS One. 2013;8;8:e72232.

5. Miller A, Pratt H, Schiffer RB. Pseudobulbar affect: the spectrum of clinical presentations, etiologies and treatments. Expert Rev Neurother. 2011. 11;7:1077-1088.

6. Andersen G, Gilbart J, Cummings J. Pseudobulbar Affect - A Disabling but Under-recognised Consequence of Neurological Disease and Brain Injury. Euro Neuro Rev. 2013;8;2:74-81.

7. Calvert T. Knapp P, House A. Psychological associations with emotionalism after stroke. J Neurol Neurosurg Psychiatry. 1998;65;6:928-929.

8. Rudolph JL, Fonda JR, Hunt PR, et al. Association of Pseudobulbar Affect symptoms with quality of life and healthcare costs in Veterans with traumatic brain injury. JAD. 2016;190:150-155.

9. Colamonico J, Formella A, Bradley W. Pseudobulbar affect: burden of illness in the USA. Adv Ther. 2012;29;9:775-798.

10. Miller RG, Jackson CE, Kasarskis EJ, et al. Practice parameter update: the care of the patient with amyotrophic lateral sclerosis: multidisciplinary care, symptom management, and cognitive/behavioral impairment (an evidence-based review): report of the Quality Standards Subcommittee of the American Academy of Neurology. Neurol. 2009;73;15:1227-1233.

11. Pioro EP. Current concepts in the pharmacotherapy of pseudobulbar affect. Drugs. 2011;71;9:11931207.

12. Choi-Kwon S, Han SW, Kwon SU, et al. Fluoxetine treatment in poststroke depression, emotional incontinence, and anger proneness: a double-blind, placebo-controlled study. Stroke. 2006;37;1:156161.

13. Pioro EP. Review of Dextromethorphan $20 \mathrm{mg} /$ Quinidine $10 \mathrm{mg}(\operatorname{NUEDEXTA}((\mathrm{R})))$ for Pseudobulbar Affect. Neurol Ther. 2014;3;1:15-28.

14. Minden SL, Feinstein A, Kalb RC, et al. Evidence-based guideline: Assessment and management of psychiatric disorders in individuals with MS: Report of the Guideline Development Subcommittee of the American Academy of Neurology. Neur. 2014;82;2: 174-181.

15. Burke NJ, Bird J., Clark MA., et al. Social and cultural meanings of self-efficacy. Health Educ Behav. 2009;36;5 Suppl:111S-128S. 
16. Moore DE, Green JS, Gallis HA. Achieving desired results and improving outcomes: integrating planning and assessment throughout learning activities. JCEHP. 2009;29;1:1-15.

17. Braun V, Clark V. Successful Qualitative Research. London: Sage; 2015.

18. Pope C, Ziebland S, Mays N. Qualitative research in health care. Analysing qualitative data. BMJ. 2000;320:114-116.

19. Cohen J. Statistical Power Analysis for the Behavioral Sciences. Mahwah, New Jersey: Lawrence Erlbaum Associations, Publisher; 1998.

20. Moore SR, Gresham LS, Bromberg MB, et al. A self report measure of affective lability. J Neurol Neurosurg Psychiatry. 1997;63;1:89-93.

21. Smith RA, Berg JE, Pope LE, et al. Validation of the CNS emotional lability scale for pseudobulbar affect (pathological laughing and crying) in multiple sclerosis patients. Multiple Sclerosis. 2004;10;6:679-685.

22. Cervero RM, Gaines JK. Effectiveness of Continuing Medical Education: Updated Synthesis of Systematic Reviews. Chicago, IL: Accreditation Council for Continuing Medical Education; 2015.

23. Cook DA, Levinson AJ, Garside S, et al. Internet-based learning in the health professions: a metaanalysis. JAMA. 2008.300;10:1181-1196.

24. Salter SM, Karia A, Sanfilippo FM, Clifford RM. Effectiveness of E-learning in pharmacy education. Am J Pharm Educ. 2014;78;4:83.

25. Salinas GD. CME effectiveness: utilizing outcomes assessments of $600+$ CME programs to evaluate the association between format and effectiveness. J Contin Educ Health Prof. 2015;35 Suppl 1:S3839.

\section{Tables}

Table 1. Interview Sample Characteristics 


\begin{tabular}{|c|c|c|}
\hline Variable & $n$ & $\%$ \\
\hline \multicolumn{3}{|l|}{ Age Range $($ mean $=50)$} \\
\hline $25-34$ & 4 & 20 \\
\hline $45-54$ & 9 & 45 \\
\hline $55-64$ & 5 & 25 \\
\hline$\geq 65$ & 2 & 10 \\
\hline \multicolumn{3}{|l|}{ Gender } \\
\hline Male & 10 & 50 \\
\hline Female & 10 & 50 \\
\hline \multicolumn{3}{|l|}{ Specialty } \\
\hline Internal medicine & 5 & 25 \\
\hline Family/General Practice & 5 & 25 \\
\hline Geriatric medicine & 2 & 10 \\
\hline Neurology & 5 & 25 \\
\hline Psychiatry & 3 & 15 \\
\hline \multicolumn{3}{|l|}{ Practice Type } \\
\hline Private solo & 9 & 45 \\
\hline Group practice & 7 & 35 \\
\hline Community (inc. hospice, federal facility) & 3 & 15 \\
\hline Academic & 1 & 5 \\
\hline \multicolumn{3}{|l|}{ Number of PBA Patients } \\
\hline $1-5$ & 10 & 50 \\
\hline $6-10$ & 3 & 15 \\
\hline $11-15$ & 2 & 10 \\
\hline$\geq 16$ & 5 & 25 \\
\hline \multicolumn{3}{|l|}{ Ethnicity } \\
\hline Non-Hispanic White or Euro-American & 14 & 70 \\
\hline Latino or Hispanic American & 2 & 10 \\
\hline Asian or Asian American (inc. East, South) & 3 & 15 \\
\hline Black, Afro-Caribbean, or African American & 1 & 5 \\
\hline \multicolumn{3}{|l|}{ Region } \\
\hline Northeast & 6 & 30 \\
\hline Southeast & 4 & 20 \\
\hline Midwest & 6 & 30 \\
\hline South & 2 & 10 \\
\hline Pacific & 2 & 10 \\
\hline
\end{tabular}


Table 2. Participant Prevalence Estimation vs PRISM Prevalence ${ }^{4}$

\begin{tabular}{|l|l|l|l|}
\hline \multicolumn{2}{|l|}{ Participant Responses } & \multicolumn{2}{l|}{ PRISM Study } \\
\hline TBI & $55 \%$ & TBI & $52.4 \%$ \\
\hline AD/PD & $50 \%$ & MS & $44.8 \%$ \\
\hline Stroke & $40 \%$ & ALS & $44.8 \%$ \\
\hline MS & $35 \%$ & Stroke & $37.8 \%$ \\
\hline ALS & $20 \%$ & AD & $29.3 \%$ \\
\hline \multicolumn{2}{|l|}{} & PD & $26 \%$ \\
\hline
\end{tabular}

Table 3. Characteristics that Differentiate PBA from Other Conditions

\begin{tabular}{|c|c|}
\hline $\begin{array}{l}\text { Spontaneous or } \\
\text { Exaggerated } \\
\text { Emotions (65\%) }\end{array}$ & $\begin{array}{l}\text { - It may be lumped into a spectrum of symptoms, but there has to be the } \\
\text { involuntary crying or laughing.[Provider 10] } \\
\text { - I think just the uncontrollable, spontaneous uncontrolled laughing or crying. } \\
\text { [Provider 2] } \\
\text { - What I learned from this webinar is that these are stereotypical, that they tend to } \\
\text { be brief, that they have no relation to the patient's moods. [Provider 3] } \\
\text { - The frequent episodic crying or laughing when it's out of context to what's going } \\
\text { on.[Provider 11] }\end{array}$ \\
\hline $\begin{array}{l}\text { Symptom duration } \\
(25 \%)\end{array}$ & $\begin{array}{l}\text { - The depression lasts for a longer period of time whereas the PBA symptoms are } \\
\text { totally inappropriate to the situation, very quick onset and then it shuts off very } \\
\text { quickly.[Provider 15] } \\
\text { - If the patient reported upon history-taking that they had some longer-term } \\
\text { depression, decrease in mood for longer time, that the tearfulness may continue } \\
\text { for hours or days, things like that.[Provider 8] }\end{array}$ \\
\hline $\begin{array}{l}\text { Presence of } \\
\text { Underlying } \\
\text { Neurologic } \\
\text { Condition (10\%) }\end{array}$ & $\begin{array}{l}\text { - Usually it's an injury to the head.[Provider 1] } \\
\text { - What would tip us is that there would be an underlying neurodegenerative } \\
\text { diagnosis. So if a person, for example, has longstanding MS, that's difficult to } \\
\text { miss.[Provider 18] } \\
\text { - It would be the crying episodes that would be that and having a past medical } \\
\text { history of some kind of neurological condition and it might lead to making me } \\
\text { think that it's not just straight depression.[Provider 5] }\end{array}$ \\
\hline
\end{tabular}

Table 4. Clinical Practice Changes Resulting from Activity Participation 


\begin{tabular}{|l|c|}
\hline $\begin{array}{l}\text { I am better able to recognize the clinical features and manifestations of pseudobulbar affect (PBA) } \\
\text { supporting its diagnosis in patients with neurologic disease. }\end{array}$ & $78 \%$ \\
\hline I have a greater appreciation for the burden of disease that may be associated with PBA. & $72 \%$ \\
\hline I am better able to differentiate PBA from depression thus avoiding misdiagnosis. & $63 \%$ \\
\hline I am better able to utilize assessment scales in patients with PBA. & $47 \%$ \\
\hline I am more cognizant of the association between PBA and traumatic brain injury. & $55 \%$ \\
\hline $\begin{array}{l}\text { I am better able to differentiate and assess the risk and benefits of treatment options for patients with } \\
\text { PBA. }\end{array}$ & $53 \%$ \\
\hline $\begin{array}{l}\text { I am better able to educate patients with PBA on the potential benefits of dextromethorphan/quinidine } \\
\text { treatment. }\end{array}$ & $52 \%$ \\
\hline $\begin{array}{l}\text { I am more knowledgeable and cognizant of potential etiologies of PBA and the prevalence of PBA in } \\
\text { neurologic disease. }\end{array}$ & $60 \%$ \\
\hline
\end{tabular}

Table 5. Changes in Knowledge and Competence

\begin{tabular}{|c|c|c|}
\hline Question Topics & $\begin{array}{l}\text { Relative change from baseline } \\
\text { to immediate posttest }\end{array}$ & $\begin{array}{l}\text { Relative change from baseline } \\
\text { to 8-week follow-up }\end{array}$ \\
\hline $\begin{array}{l}\text { Patient with stroke and identification of } \\
\text { signs and symptoms of PBA }\end{array}$ & $26 \% \uparrow(p<0.001 ; d=0.509)$ & $22 \% \uparrow(p=0.027 ; d=0.488)$ \\
\hline $\begin{array}{l}\text { Patient with newly diagnosed PBA and } \\
\text { appropriate treatment }\end{array}$ & $131 \% \uparrow(p=0.273)$ & $136 \% \uparrow(p=0.001 ; d=1.608)$ \\
\hline $\begin{array}{l}\text { Relationship between PBA and social } \\
\text { phobia, withdrawal and isolation }\end{array}$ & $42 \% \uparrow(p=0.001 ; d=0.610)$ & $32 \% \downarrow(p=0.012 ; d=0.495)$ \\
\hline $\begin{array}{l}\text { Relationship between PBA and various } \\
\text { other medical conditions }\end{array}$ & $32 \% \uparrow(p<0.001 ; d=0.689)$ & $13 \% \uparrow(p=0.115)$ \\
\hline
\end{tabular}

\section{Figures}




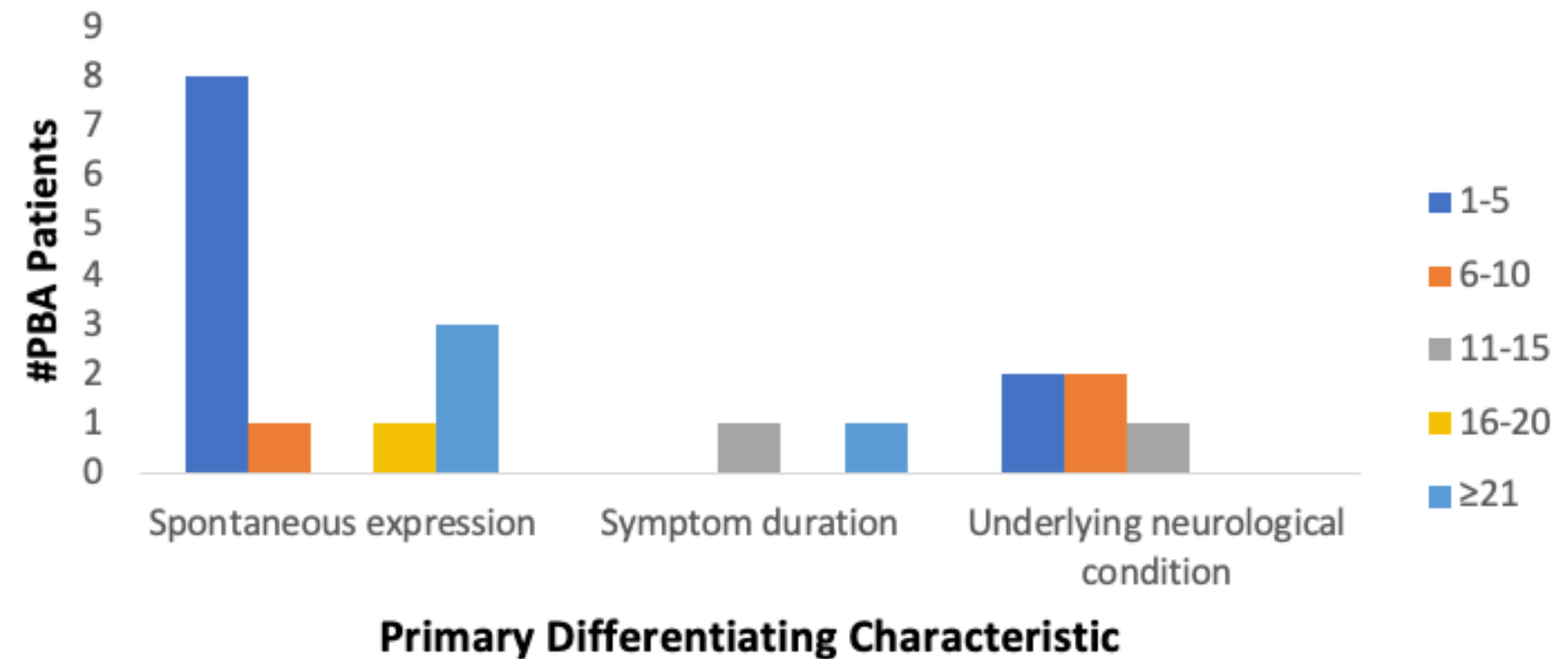

Figure 1

Differentiating Characteristics of PBA by Number of PBA Patients

\section{Supplementary Files}

This is a list of supplementary files associated with this preprint. Click to download.

- AdditionalFiles.docx 